\title{
Inferring reasons for the failure of Staphylococcus aureus vaccines in clinical trials
}

\section{Fabio Bagnoli*, Sylvie Bertholet and Guido Grandi}

Novartis Vaccines and Diagnostics, Siena, Italy

*Correspondence: fabio.bagnoli@novartis.com

Multidrug resistant Staphylococcus aureus strains are emerging and current antibiotics are not efficacious against such strains. Therefore, there is an urgent need to develop vaccines to target this pathogen. However, both active and passive immunization strategies have thus far failed to show efficacy in humans. There are several potential reasons behind the disappointing results of clinical trials, however, we believe that just a few of them, which are common to all the trials, determined their downfall. First of all, preclinical results obtained with antigens tested in clinical trials were likely overestimated by vaccine manufacturers. Furthermore, vaccines tested in humans to date, since they all targeted single antigens, were probably disproportionate to the complex pathogenic mechanisms of the bacterium. In addition, the lack of known correlates of protection in humans has severely limited the ability to interpret both preclinical and clinical data. Finally, the vaccines did not contain new generation adjuvants, which may be critical in augmenting antibody production and steering the T-cell response toward the proper profile of cytokine production.

\section{ANTIGENS TESTED IN CLINICAL TRIALS GENERATE ONLY PARTIAL PROTECTION IN ANIMAL MODELS}

\footnotetext{
We often read in recent publications the statement: "the vaccine failed in clinical trials despite being protective against staphylococcal infection in animal models."
}

We think that this parallel should not be made.

Staphylococcus aureus capsular polysaccharides type 5 and 8 (CP5 and 8) conjugated with recombinant Pseudomonas aeruginosa exoprotein A were included in the first vaccine tested in humans, StaphVax (by Nabi Biopharmaceutical), which failed in phase III clinical trial in 2005. No consistent pro- tective efficacy appears to be associated with CP8 immunization (Tuchscherr et al., 2008; Cook et al., 2009), and this may have significantly contributed to the clinical failure of StaphVax. Indeed, CP8 is expressed by approximately $40 \%$ of the circulating strains and therefore the poor protective efficacy associated with this antigen could have significantly affected the trials. On the other hand, although vaccination with CP5 has been shown to be efficacious in animal models of staphylococcal infection, $S$. aureus USA300, one of the most important clones worldwide, has recently been found to elaborate no detectable capsule because of a point mutation in the promoter region of cap-5, necessary for CP5 biosynthesis (Montgomery et al., 2008). Furthermore, the role of CPs in S. aureus virulence is not clear and their expression is limited to the stationary phase (O'Riordan and Lee, 2004; Schaffer and Lee, 2008).

Given all these variables, we think that the only approach to reliably measure vaccine efficacy in animal models is to perform challenge experiments with a large collection of strains representative of the circulating staphylococcal clones, and use different models with different routes of infection as well as low and large bacterial inocula. By doing that, vaccine efficacy will be tested in a number of different conditions, some of which may be more favorable than others. Ideally, a vaccine should consistently protect in all conditions.

A second failure occurred with an active immunization strategy based on a single surface staphylococcal protein, IsdB (Kuklin et al., 2006; Spellberg and Daum, 2011). The phase II/III vaccine clinical trial was interrupted, however, the precise reason for its premature interruption is not yet completely understood (Spellberg and Daum, 2011). IsdB is a protein which mediates iron uptake from hemoglobin. The protein shows several favorable characteristics as a vaccine candidate. Indeed, IsdB is con- served and expressed throughout different S. aureus strains and is an important virulence factor (Kuklin et al., 2006; Kim et al., 2010). However, as an antigen, the protein generated only partial protection in lethal infection mouse models (Kuklin et al., 2006; Stranger-Jones et al., 2006).

On top of active immunization, a number of passive approaches have also been undertaken: Veronate, based on polyclonal antibodies against the $S$. aureus surface protein ClfA; Altastaph, containing CP5 and CP8 antibodies purified from subjects vaccinated with StaphVax; Tefibazumab, monoclonal antibodies against ClfA; and Aurograb, single chain antibodies against an $\mathrm{ABC}$ transporter of the pathogen. They all failed to show efficacy against $S$. aureus infection in humans (Schaffer and Lee, 2008; Ohlsen and Lorenz, 2010; Otto, 2010).

The vaccines tested so far in humans differ quite substantially in the nature of the immunogen used, the immunization approach and the target population. However, there is an obvious commonality between them: they all target a single component of the pathogen. Since S. aureus expresses a plethora of toxins and immune evasion factors, efficacy of monovalent vaccines is likely to be insufficient in humans and this is reflected by partial protection achieved in animal models.

\section{VACCINES SO FAR TESTED IN CLINICAL TRIALS ARE NOT THE BEST AVAILABLE OPTION}

We strongly believe that a multivalent vaccine is needed against $S$. aureus. Indeed, it has already been demonstrated that protein combinations can generate additive protection (Stranger-Jones et al., 2006; Kim et al., 2011). In particular a combination of four surface proteins (IsdB, IsdA, SdrD, and $\mathrm{SdrE}$ ) was shown to protect mice from lethal infection with $S$. aureus Newman strain with greater efficacy than any of the single components (Stranger-Jones et al., 
2006). Importantly, in this model IsdB, the candidate tested by Merck in clinical trials, failed to generate protective immunity. The additive efficacy may be due to the different functions played by the four antigens in $S$. aureus virulence. This hypothesis is supported by the observation that a $S$. aureus SrtA knockout mutant, which lacks all the LPXTG-containing proteins (including IsdB, IsdA, SdrD, and SdrE), is less virulent than any mutant of individual LPXTG proteins (Cheng et al., 2009). Stranger-Jones et al. (2006) also tested the protective efficacy of several other $S$. aureus antigens in a murine renal abscess model in terms of bacterial load reduction. ClfA, the target of two clinical trials based on passive immunization, was found to be inferior to IsdB. Therefore, it is conceivable to assume that strategies targeting ClfA only, may not be sufficiently protective. In a more recent paper two protein combinations (ClfA, FnBPB, and SdrD) and (ClfA, FnBPB, $\mathrm{SdrD}$, and a non-toxigenic form of protein A, SpAKKAA) were shown to be highly protective in two different animal models of staphylococcal infection (Kim et al., 2011). Interestingly, the four-component combination elicited greater protection than the trivalent vaccine. Similar results were observed with passive immunization studies, such as the antibody combination of ClfA and FnBPA (Arrecubieta et al., 2008). Our internal research with several antigens known in the literature as well as novel candidates confirms that, in general, combinations generate greater protection than single antigens (unpublished data).

\section{PROTECTIVE MECHANISMS AGAINST S. AUREUS REMAIN TO BE ELUCIDATED}

The lack of correlates of protection for $S$. aureus is one of the major challenges for vaccine design and development. The failures of passive immunization strategies in clinical trials, advocate against an important role for antibodies in mediating protection against $S$. aureus. However, we believe that both preclinical and clinical data suggest that humoral responses play, at least, a partial role in protecting against the pathogen. Passive transfer of antibodies raised against different staphylococcal antigens (e.g., $\mathrm{Hla}$, IsdA, IsdB) in animal models, confers protection against the infection (Bubeck Wardenburg and Schneewind, 2008; Kim et al., 2010). On the other hand, in vitro assays have shown that antibodies can have a direct role in inhibiting the function of virulence factors and toxins. For example, antibodies can neutralize the toxicity of Hla or interfere with heme-iron scavenging mediated by IsdB and IsdA (Bubeck Wardenburg and Schneewind, 2008; Kim et al., 2010). Moreover, antibodies against several antigens have been shown to mediate opsonophagocytosis (Stranger-Jones et al., 2006). From the clinical point of view, the scenario is much less clear. It is very well know that most individuals have circulating antibodies to S. aureus (Dryla et al., 2005; Clarke et al., 2006; Verkaik et al., 2009), however it is not known if they confer any protective immunity (Hermos et al., 2010). In our mind, an indication that antibodies do play, at least, a partial role in protecting humans against severe staphylococcal infections, comes from the observation that colonized patients present milder disease outcomes as compared to non-colonized patients (Wertheim et al., 2004). Indeed, colonized subjects have been shown to have higher antibodies titers against several staphylococcal antigens (Verkaik et al., 2009). Finally, sera from vaccines of StaphVax trials were found to mediate opsonophagocytosis and human sera of volunteers immunized with CP5 to confer passive protection in a murine infection model (Fattom et al., 1996, 2004). As already discussed for active vaccination strategies, failure of trials conducted with passive immunization may be due to the insufficient efficacy achieved targeting single antigens. In addition, immune evasion mechanisms deployed by $S$. aureus, may abolish or dampen anti-S. aureus humoral responses in humans (Serruto et al., 2010a; Kim et al., 2012).

\section{S. AUREUS IMMUNE EVASION FACTORS MAY REPRESENT A MAJOR CHALLENGE IN DEVELOPING EFFICACIOUS VACCINES}

S. aureus expresses a plethora of factors which, in different manners, inhibit the host immune response against the pathogen. Among them, the IgG-binding proteins SpA and Sbi, and complement-binding factors have been shown to efficiently interfere with antibody-mediated protection mechanisms (Serruto et al., 2010a; Kim et al., 2012). A critical aspect of some of these factors is the human specificity. For example SCIN, CHIPS, and SAK, which directly or indirectly inhibit $\mathrm{C} 3$ convertase, $\mathrm{C} 5 \mathrm{a}$ receptor, and $\mathrm{C} 3 \mathrm{~b}$, respectively, are all human specific (Rooijakkers et al., 2005; Serruto et al., 2010b). It is therefore likely that the contribution of these factors in evading the host immune response is underestimated in animal models. Therefore, protective efficacy of vaccines tested in animals may generally be greater than in humans. Development of improved animal models, such as humanized mice, and reliable surrogates of protection, such as the opsonophagocytosis assay, is another critical aspect that the scientific community needs to address to increase the likelihood of success of S. aureus vaccines. On the other hand, the presence of such mechanisms indicates that antibodymediated protection is potentially important against $S$. aureus. Therefore, to develop efficacious vaccines we need to understand how to avoid or compensate for the detrimental effect of complement evasion factors on the host immune response.

\section{CELL-MEDIATED IMMUNITY MAY BE A KEY ELEMENT OF S. AUREUS VACCINES}

Patients with disruption of anatomical barriers, and those with quantitative and qualitative T-cell or neutrophil disorders are definitively at increased risk of developing staphylococcal infections (Spellberg and Daum, 2011).

These observations were recapitulated in animal models demonstrating that B celldeficient mice were no more susceptible to systemic infection caused by $S$. aureus compared with wild-type mice (Spellberg et al., 2008), while Tcell-deficient (Spellberg et al., 2008), IFN- $\boldsymbol{\gamma}$-deficient (Spellberg et al., 2008; Lin et al., 2009), TNF-deficient (Hultgren et al., 1998), and dual IL-17A/Fdeficient (Ishigame et al., 2009) mice were hypersusceptible to $S$. aureus infection. Furthermore, Th17 cells were necessary for vaccine-induced protection against S. aureus infection by enhancing neutrophil recruitment to sites of infection, and killing of the bacteria (Lin et al., 2009). Superoxide-deficient mice (a model of chronic granulomatous disease) were also more susceptible to infection. Altogether, these data indicate that protection against $S$. aureus infections requires intact phagocytic function and is markedly enhanced by 
Th1/Th17 adaptive immunity, suggesting that cell-mediated focused vaccines could effectively protect against $S$. aureus infections.

\section{ADJUVANTS MAY BE IMPORTANT TO STEER THE RIGHT RESPONSE AGAINST S. AUREUS}

Traditionally, adjuvants have been used to increase antibody-mediated responses. Aluminum hydroxyde (alum), oil-in-water emulsions (e.g., MF59 and AS03), have been shown to increase antibody titers, effectiveness, and even functionality (Galli et al., 2009; Jones, 2009; Dormitzer et al., 2011). However, the important role of adjuvants in stimulating T-cell responses is also becoming clear. Recently, the response generated by MF59 was demonstrated to be skewed toward Th1 by the addition of E6020, a synthetic analog of MPL (monophosphoryl lipid A; Baudner et al., 2009; Dormitzer et al., 2011). In another study, IC31, a KLK peptide plus non-CpG oligonucleotide, was demonstrated to, conversely to alum, induce strong Th1 and Th17 responses (Kamath et al., 2008). Therefore, on top of the proper antigen combination, design of adjuvant formulations inducing higher and more functional antibodies, and stimulating T-cell-mediated immunity will certainly be another critical area of investigation.

\section{CONCLUSION}

In our opinion, the spreading fear that an efficacious vaccine against $S$. aureus is not feasible, is not justified. Doubts expressed by several scientists are obviously due to the numerous failures in clinical trials and to the lack of known correlates of protection in humans (Patti, 2011; Proctor, 2012; Spellberg and Daum, 2011). Furthermore, concerns are also based on the observation that failed vaccines were assumed to be efficacious in animal models. However, vaccines tested in humans so far generated only partial protection against staphylococcal infection in animal models. All vaccines tested in clinical trials targeted a single $S$. aureus component. Studies using antigen combinations have shown greater efficacy than single antigen vaccines in animal models. Therefore, multivalent vaccines will likely work better in humans as well. At the same time, we should be cautious in making direct correlations between animal studies and clinical trials. Indeed, although several

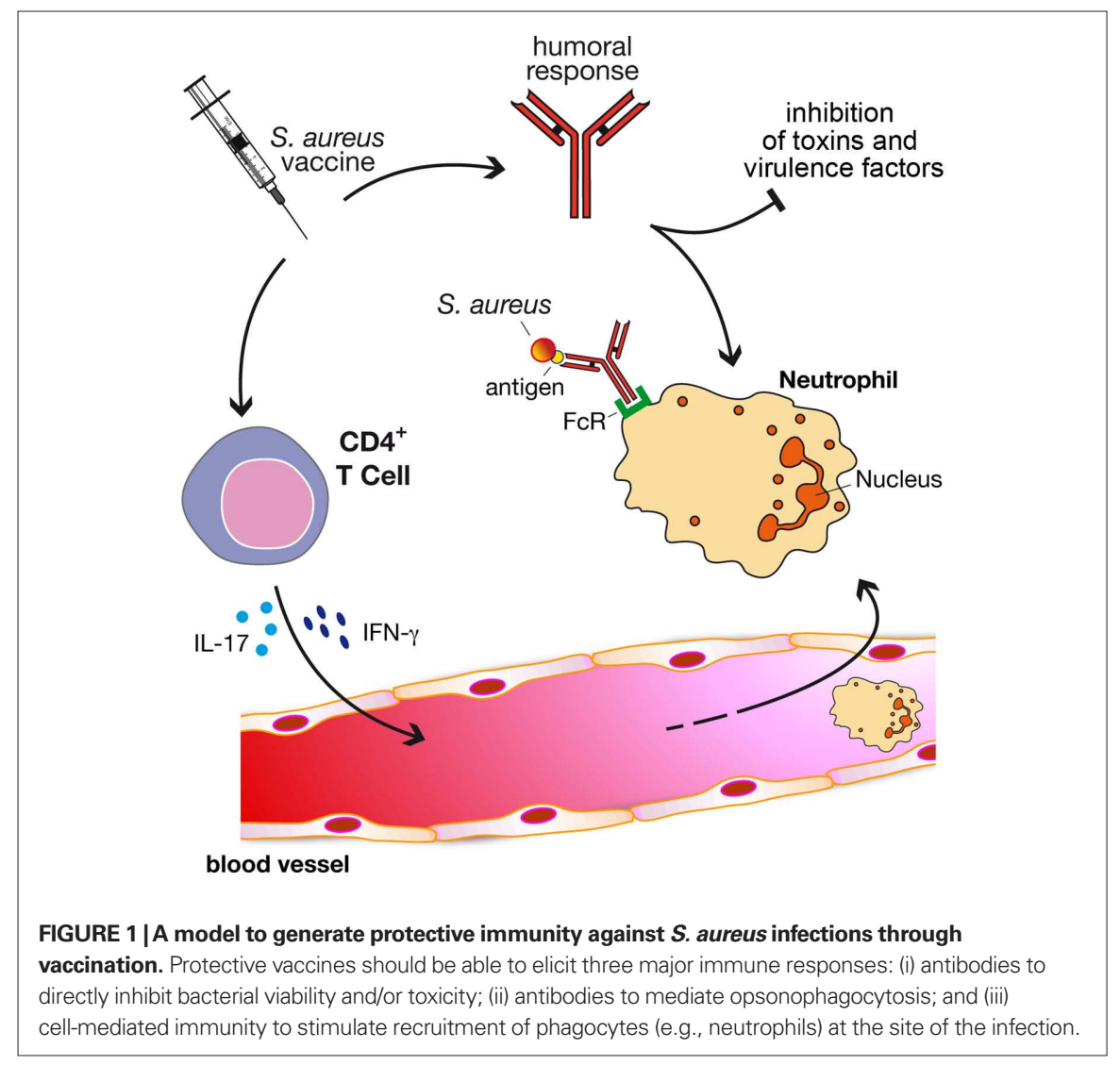

animal infection models have been established for assessing preclinical protective efficacy of vaccines, they may not optimally resemble natural infections in humans.

Accumulating literature is unraveling S. aureus pathogenic and immune evasion mechanisms. It will be important to use this information to target critical factors involved in these processes with next generation vaccines and to develop improved animal models and surrogates of protection. Although the nature of protective immunity against the pathogen in humans is not known, animal studies as well as clinical observations indicate that both humoral as well as cell-mediated immunity are involved.

On the basis of that, we propose a model (Figure 1) in which vaccine efficacy is gained through three major immune responses: (i) antibodies to directly inhibit bacterial viability and/or toxicity; (ii) antibodies to mediate opsonophagocytosis; and (iii) cell-mediated immunity to stimulate recruitment of phagocytes at the site of the infection. It is very likely that only a combination of staphylococcal antigens with different properties and functions formulated with adjuvants able to elicit a potent antibody production, but also the proper cellular response, will satisfy the three criteria.

In conclusion, we are confident that by performing extensive preclinical work, using different animal models, readouts, and challenge strains, S. aureus vaccines clinical trials can be developed.

\section{ACKNOWLEDGMENTS}

We are grateful to Prof. Manuel Amieva (Stanford University) for critical reading of the paper and to our colleague Giorgio Corsi for art work.

\section{REFERENCES}

Arrecubieta, C., Matsunaga, I., Asai, T., Naka, Y., Deng, M. C., and Lowy, F. D. (2008). Vaccination with clumping factor A and fibronectin binding protein A to prevent Staphylococcus aureus infection of an aortic patch in mice. J. Infect. Dis. 198, 571-575.

Baudner, B. C., Ronconi, V., Casini, D., Tortoli, M., Kazzaz, J., Singh, M., Hawkins, L. D., Wack, A., and O'Hagan, D. T. (2009). MF59 emulsion is an effective delivery system for a synthetic TLR4 agonist (E6020). Pharm. Res. 26, 1477-1485. with much greater chances of success in 
Bubeck Wardenburg, J., and Schneewind, O. (2008). Vaccine protection against Staphylococcus aureus pneumonia. J. Exp. Med. 205, 287-294.

Cheng, A. G., Kim, H. K., Burts, M. L., Krausz, T., Schneewind, O., and Missiakas, D. M. (2009). Genetic requirements for Staphylococcus aureus abscess formation and persistence in host tissues. FASEB J. 23, 3393-3404.

Clarke, S. R., Brummell, K. J., Horsburgh, M. J., Mcdowell, P. W., Mohamad, S. A., Stapleton, M. R., Acevedo, J., Read, R. C., Day, N. P., Peacock, S. J., Mond, J. J., Kokai-Kun, J. F., and Foster, S. J. (2006). Identification of in vivo-expressed antigens of Staphylococcus aureus and their use in vaccinations for protection against nasal carriage. J. Infect. Dis. 193, 1098-1108.

Cook, J., Hepler, R., Pancari, G., Kuklin, N., Fan, H., Wang, X. M., Cope, L., Tan, C., Joyce, J., Onishi, J., Montgomery, D., Anderson, A., and McNeely, T. (2009). Staphylococcus aureus capsule type 8 antibodies provide inconsistent efficacy in murine models of staphylococcal infection. Hum. Vaccin. 5, 254-263.

Dormitzer, P. R., Galli, G., Castellino, F., Golding, H., Khurana, S., Del Giudice, G., and Rappuoli, R. (2011). Influenza vaccine immunology. Immunol. Rev. 239, 167-177.

Dryla, A., Prustomersky, S., Gelbmann, D., Hanner, M., Bettinger, E., Kocsis, B., Kustos, T., Henics, T., Meinke, A., and Nagy, E. (2005). Comparison of antibody repertoires against Staphylococcus aureus in healthy individuals and in acutely infected patients. Clin. Diagn. Lab. Immunol. 12, 387-398.

Fattom, A. I., Horwith, G., Fuller, S., Propst, M., and Naso, R. (2004). Development of StaphVAX, a polysaccharide conjugate vaccine against $S$. aureus infection: from the lab bench to phase III clinical trials. Vaccine 22, 880-887.

Fattom, A. I., Sarwar, J., Ortiz, A., and Naso, R. (1996). A Staphylococcus aureus capsular polysaccharide (CP) vaccine and CP-specific antibodies protect mice against bacterial challenge. Infect. Immun. 64, 1659-1665.

Galli, G., Hancock, K., Hoschler, K., Devos, J., Praus, M., Bardelli, M., Malzone, C., Castellino, F., Gentile, C., Mcnally, T., Del Giudice, G., Banzhoff, A., Brauer, V., Montomoli, E., Zambon, M., Katz, J., Nicholson, K., and Stephenson, I. (2009). Fast rise of broadly crossreactive antibodies after boosting long-lived human memory B cells primed by an MF59 adjuvanted prepandemic vaccine. Proc. Natl. Acad. Sci. U.S.A. 106, 7962-7967.

Hermos, C. R., Yoong, P., and Pier, G. B. (2010). High levels of antibody to panton-valentine leukocidin are not associated with resistance to Staphylococcus aureus-associated skin and soft-tissue infection. Clin. Infect. Dis. 51, 1138-1146.

Hultgren, O., Eugster, H. P., Sedgwick, J. D., Korner, H., and Tarkowski, A. (1998). TNF/lymphotoxin-alpha double-mutant mice resist septic arthritis but display increased mortality in response to Staphylococcus aureus. J. Immunol. 161, 5937-5942.

Ishigame, H., Kakuta, S., Nagai, T., Kadoki, M., Nambu, A., Komiyama, Y., Fujikado, N., Tanahashi, Y., Akitsu, A., Kotaki, H., Sudo, K., Nakae, S., Sasakawa, C., and Iwakura, Y. (2009). Differential roles of interleukin-
17A and -17F in host defense against mucoepithelial bacterial infection and allergic responses. Immunity 30, 108-119.

Jones, T. (2009). GSK's novel split-virus adjuvanted vaccines for the prevention of the $\mathrm{H} 5 \mathrm{~N} 1$ strain of avian influenza infection. Curr. Opin. Mol. Ther. 11, 337-345.

Kamath, A. T., Rochat, A. F., Valenti, M. P., Agger, E. M., Lingnau, K., Andersen, P., Lambert, P. H., and Siegrist, C. A. (2008). Adult-like anti-mycobacterial $\mathrm{T}$ cell and in vivo dendritic cell responses following neonatal immunization with Ag85B-ESAT-6 in the IC31 adjuvant. PLoS ONE 3, e3683. doi: 10.1371/ journal.pone.0003683

Kim, H. K., Dedent, A., Cheng, A. G., Mcadow, M., Bagnoli, F., Missiakas, D. M., and Schneewind, O. (2010). Isd A and IsdB antibodies protect mice against Staphylococcus aureus abscess formation and lethal challenge. Vaccine 28, 6382-6392.

Kim, H. K., Kim, H. Y., Schneewind, O., and Missiakas, D. (2011). Identifying protective antigens of Staphylococcus aureus, a pathogen that suppresses host immune responses. FASEB J. 25, 3605-3612.

Kim, H. K., Thammavongsa, V., Schneewind, O., and Missiakas, D. (2012). Recurrent infections and immune evasion strategies of Staphylococcus aureus. Curr. Opin. Microbiol. 15, 92-99.

Kuklin, N. A., Clark, D. J., Secore, S., Cook, J., Cope, L. D., Mcneely, T., Noble, L., Brown, M. J., Zorman, J. K., Wang, X. M., Pancari, G., Fan, H., Isett, K., Burgess, B., Bryan, J., Brownlow, M., George, H., Meinz, M., Liddell, M. E., Kelly, R., Schultz, L., Montgomery, D., Onishi, J., Losada, M., Martin, M., Ebert, T., Tan, C. Y., Schofield, T. L., Nagy, E., Meineke, A., Joyce, J. G., Kurtz, M. B., Caulfield, M. J., Jansen, K. U., Mcclements, W., and Anderson, A. S. (2006). A novel Staphylococcus aureus vaccine: iron surface determinant B induces rapid antibody responses in rhesus macaques and specific increased survival in a murine $S$. aureus sepsis model. Infect. Immun. 74, 2215-2223.

Lin, L., Ibrahim, A. S., Xu, X., Farber, J. M., Avanesian, V., Baquir, B., Fu, Y., French, S. W., Edwards, J. E. Jr., and Spellberg, B. (2009). Th1-Th17 cells mediate protective adaptive immunity against Staphylococcus aureus and Candida albicans infection in mice. PLoS Pathog. 5, e1000703. doi: 10.1371/journal. ppat. 1000703

Montgomery, C. P., Boyle-Vavra, S., Adem, P. V., Lee, J. C., Husain, A. N., Clasen, J., and Daum, R. S. (2008). Comparison of virulence in community-associated methicillin-resistant Staphylococcus aureus pulsotypes USA300 and USA400 in a rat model of pneumonia. J. Infect. Dis. 198, 561-570.

Ohlsen, K., and Lorenz, U. (2010). Immunotherapeutic strategies to combat staphylococcal infections. Int. J. Med. Microbiol. 300, 402-410.

O'Riordan, K., and Lee, J. C. (2004). Staphylococcus aureus capsular polysaccharides. Clin. Microbiol. Rev. 17, 218-234.

Otto, M. (2010). Novel targeted immunotherapy approaches for staphylococcal infection. Expert Opin. Biol. Ther. 10, 1049-1059.

Patti, J. M. (2011). Will we ever see the approval of a Staphylococcus aureus vaccine? Expert Rev. Anti. Infect. Ther. 9, 845-846.
Proctor, R. (2012). Is there a future for a Staphylococcus aureus vaccine? Vaccine (in press).

Rooijakkers, S. H., Van Kessel, K. P., and Van Strijp, J. A. (2005). Staphylococcal innate immune evasion. Trends Microbiol. 13, 596-601.

Schaffer, A. C., and Lee, J. C. (2008). Vaccination and passive immunisation against Staphylococcus aureus. Int. J. Antimicrob. Agents 32(Suppl. 1), S71-S78.

Serruto, D., Rappuoli, R., Scarselli, M., Gros, P., and Van Strijp, J. A. (2010a). Molecular mechanisms of complement evasion: learning from staphylococci and meningococci. Nat. Rev. Microbiol. 8, 393-399.

Serruto, D., Spadafina, T., Ciucchi, L., Lewis, L.A., Ram, S., Tontini, M., Santini, L., Biolchi, A., Seib, K. L., Giuliani, M. M., Donnelly, J. J., Berti, F., Savino, S., Scarselli, M., Costantino, P., Kroll, J. S., O’Dwyer, C., Qiu, J., Plaut, A. G., Moxon, R., Rappuoli, R., Pizza, M., and Arico, B. (2010b). Neisseria meningitidis GNA2132, a heparinbinding protein that induces protective immunity in humans. Proc. Natl. Acad. Sci. U.S.A. 107, 3770-3775.

Spellberg, B., and Daum, R. (2012). Development of a vaccine against Staphylococcus aureus. Semin. Immunopathol. 34, 335-348.

Spellberg, B., Ibrahim, A. S., Yeaman, M. R., Lin, L., Fu, Y., Avanesian, V., Bayer, A. S., Filler, S. G., Lipke, P., Otoo, H., and Edwards, J. E. Jr. (2008). The antifungal vaccine derived from the recombinant $\mathrm{N}$ terminus of Als $3 p$ protects mice against the bacterium Staphylococcus aureus. Infect. Immun. 76, 4574-4580.

Stranger-Jones, Y. K., Bae, T., and Schneewind, O. (2006). Vaccine assembly from surface proteins of Staphylococcus aureus. Proc. Natl. Acad. Sci. U.S.A. 103, 16942-16947.

Tuchscherr, L. P., Buzzola, F. R., Alvarez, L. P., Lee, J. C., and Sordelli, D. O. (2008). Antibodies to capsular polysaccharide and clumping factor A prevent mastitis and the emergence of unencapsulated and small-colony variants of Staphylococcus aureus in mice. Infect. Immun. 76, 5738-5744.

Verkaik, N. J., De Vogel, C. P., Boelens, H. A., Grumann, D., Hoogenboezem, T., Vink, C., Hooijkaas, H., Foster, T. J., Verbrugh, H.A., Van Belkum, A., and Van Wamel, W. J. (2009). Anti-staphylococcal humoral immune response in persistent nasal carriers and noncarriers of Staphylococcus aureus. J. Infect. Dis. 199, 625-632.

Wertheim, H. F., Vos, M. C., Ott, A., Van Belkum, A., Voss, A., Kluytmans, J. A., Van Keulen, P. H., Vandenbroucke-Grauls, C. M., Meester, M. H., and Verbrugh, H. A. (2004). Risk and outcome of nosocomial Staphylococcus aureus bacteraemia in nasal carriers versus non-carriers. Lancet 364, 703-705.

Received: 02 January 2012; accepted: 04 February 2012; published online: 22 February 2012.

Citation: Bagnoli F, Bertholet $S$ and Grandi G (2012) Inferring reasons for the failure of Staphylococcus aureus vaccines in clinical trials. Front. Cell. Inf. Microbio. 2:16. doi: 10.3389/fcimb.2012.00016

Copyright (c) 2012 Bagnoli, Bertholet and Grandi. This is an open-access article distributed under the terms of the Creative Commons Attribution Non Commercial License, which permits non-commercial use, distribution, and reproduction in other forums, provided the original authors and source are credited. 